\title{
FINITE DIMENSIONAL GROUP RINGS ${ }^{1}$
}

\author{
RALPH W. WILKERSON
}

\begin{abstract}
A ring is right finite dimensional if it contains no infinite direct sum of right ideals. We prove that if a group $G$ is finite, free abelian, or finitely generated abelian, then a ring $R$ is right finite dimensional if and only if the group ring $R G$ is right finite dimensional. A ring $R$ is a self-injective cogenerator ring if $R_{R}$ is injective and $R_{R}$ is a cogenerator in the category of unital right $R$-modules; this means that each right unital $R$-module can be embedded in a direct product of copies of $R$. Let $G$ be a finite group where the order of $G$ is a unit in $R$. Then the group ring $R G$ is a selfinjective cogenerator ring if and only if $R$ is a self-injective cogenerator ring. Additional applications are given.
\end{abstract}

1. Introduction. Let $R$ always denote an associative ring with 1 and $G$ a group with order $|G|$. The group ring of a group $G$ and a $\operatorname{ring} R$ is the ring of all formal sums $\sum_{g \in G} r(g) g$ with $r(g) \in R$ and with only finitely many nonzero $r(g)$ [7]. For a right finite dimensional ring $R$, there exists an integer $n$ such that $R$ contains a direct sum of $n$-summands and the number of summands of any other direct sum in $R$ is at most $n$. In this case, we write $\operatorname{dim} R=n$. The ring $R$ will be considered as a right $R$-module $R_{R}$ and by finite dimensional we shall mean right finite dimensional.

It is known that if $H$ is any semigroup with 1 , then $R H$ is a ring. In particular, the polynomial ring is a special case of this construction. Shock has shown that the right finite dimensional property carries over to polynomial rings [10]. This paper extends this result to group rings.

If $R$ is a subring of $Q$ and the identity of $R$ is also the identity of $Q$, then $R$ is a right order in $Q$ if

(a) every nonzero divisor of $R$ is a unit in $Q$, and

(b) every element of $Q$ can be written in the form of $c d^{-1}$ where $c$ and $d$ are in $R$ and $d$ is a nonzero divisor of $R$. We prove that if $G$ is a finite group, then $R$ is a right order in a self-injective cogenerator ring and the order of no finite normal subgroup of $G$ is a zero-divisor in $R$

Presented to the Society, January 28, 1973; received by the editors February 27, 1973. AMS (MOS) subject classifications (1970). Primary 16A26, 16A52, $20 \mathrm{C05.}$

Key words and phrases. Group ring, injective, order, cogenerator, rationally closed, dense right ideal, complete ring of quotients, right finite dimensional.

${ }^{1}$ This article is part of the author's doctoral thesis which was written under the direction of Professor Robert C. Shock.

(c) American Mathematical Society 1973 
if and only if $R G$ is a right order in a self-injective cogenerator ring. Let $G$ be a free abelian group. If $R$ is a right order in a right Artinian ring then $R G$ is a right order in a right Artinian ring.

2. Finite dimensional group rings. It is always true that if $R G$ is finite dimensional then $R$ is finite dimensional; however, the converse is not in general true.

EXAMPLE 2.1. There exists a finite dimensional ring $R$ and a group $G$ such that the group ring $R G$ is not finite dimensional. Let $R$ be a field of characteristic zero and $G=\oplus \sum_{C_{p}}$ (for all prime $p$ ), where $C_{p}$ is a cyclic group of order $p$. Then $R G$ is not finite dimensional. This follows from the fact that $R G$ is regular and the right ideal $\omega\left(C_{p}\right)$ of $R G$ generated by $\left\{1-h \mid h \in C_{p}\right\}$ is principal [2]. So the question naturally arises as to when the group ring $R G$ is finite dimensional.

Proposition 2.2 (Shock [10]). $A$ ring $R$ is finite dimensional if and only if the polynomial ring $R\left[x_{1}, x_{2}, \cdots\right]$ is finite dimensional. Furthermore, $\operatorname{dim} R=\operatorname{dim} R\left[x_{1}, x_{2}, \cdots\right]$.

Proof. See Theorem 2.6 of [10].

Let $R$ be a subring of $S$, then we call $S$ a ring of right quotients of $R$, if for every $0 \neq s \in S$ and for every $s^{\prime} \in S$, there exists $r \in R$ such that $s r \neq 0$ and $s^{\prime} r \in R$. Let $Q(R)$ denote the complete ring of quotients of $R$. It is well known that $R$ is finite dimensional if and only if $Q(R)$ is, and in this case $\operatorname{dim} R=\operatorname{dim} Q(R)$. It is also known that if $S$ is a ring of right quotients of $R$ then $Q(R)$ is the complete ring of quotients of $S$ [4].

THEOREM 2.3. Let $G$ be an infinite cyclic group, then $R$ is finite dimensional if and only if $R G$ is finite dimensional. Furthermore, $\operatorname{dim} R=\operatorname{dim} R G$.

Proof. Let $S$ be a multiplicative semigroup isomorphic to the nonnegative integers. Then $S$ is a semigroup with identity and is generated by the nonnegative powers of some element, say $g$. By Proposition 2.2, it is clear that $R S$ is finite dimensional, since $R S$ is just a polynomial ring in the variable $g$. Now $S$ can be embedded in an infinite cyclic group $G$, which is generated by all powers of $g$. We need only show that $R G$ is a ring of right quotients of $R S$. Let $r_{1}, r_{2} \in R G$ with

and

$$
\begin{aligned}
0 \neq r_{1} & =r_{1}\left(g_{1}\right) g_{1}+\cdots+r_{1}\left(g_{n}\right) g_{n} \\
& =r_{1}\left(g_{1}\right) g^{a_{1}}+\cdots+r_{1}\left(g_{n}\right) g^{a_{n}}
\end{aligned}
$$

$$
\begin{aligned}
r_{2} & =r_{2}\left(h_{1}\right) h_{1}+\cdots+r_{2}\left(h_{m}\right) h_{m} \\
& =r_{2}\left(h_{1}\right) g^{b_{1}}+\cdots+r_{2}\left(h_{m}\right) g^{b_{m}} .
\end{aligned}
$$


Let $k=\max \left\{\left|a_{i}\right|,\left|b_{j}\right|\right\}$ for all $1 \leqq i \leqq n$ and $1 \leqq j \leqq m$. It is clear that $r=g^{k} \in R S, r_{1} r \neq 0$, and $r_{2} r \in R S$. Hence, $R G$ is finite dimensional. Also, $\operatorname{dim} Q(R S)=\operatorname{dim} R S=\operatorname{dim} R$ shows that $\operatorname{dim} R=\operatorname{dim} R G$. The converse is clear.

A free abelian group is a group which is a direct sum of infinite cyclic groups.

COROLlaRY 2.4. Let $G$ be a free abelian group, then $R$ is finite dimensional if and only if $R G$ is finite dimensional. Furthermore, $\operatorname{dim} R=\operatorname{dim} R G$.

Proof. Let $H=S_{1} \oplus S_{2} \oplus \cdots$ where each $S_{i}$ is a multiplicative semigroup isomorphic to the nonnegative integers. If $R$ is finite dimensional then $R H$ is finite dimensional by Proposition 2.2. Let $G=G_{1} \oplus G_{2} \oplus \cdots$, where $S_{i}$ is embedded in the infinite cyclic group $G_{i}$, and now show that $R G$ is a ring of right quotients of $R H$. The details are omitted. The converse and $\operatorname{dim} R=\operatorname{dim} R G$ follow easily.

LEMMA 2.5. For a finite group $G$, the group ring $R G$ is finite dimensional if and only if the ring $R$ is finite dimensional. Also, $\operatorname{dim} R \leqq \operatorname{dim} R G \leqq$ $\operatorname{dim} R \cdot|G|$.

Proof. Let $G$ be finite, then $R G_{R}$ is $R$-isomorphic to a direct sum of $|G|$ copies of the finite dimensional $R$-module $R$. Hence, $R G$ is a finite dimensional $R$-module and therefore a finite dimensional $R G$-module. The converse and inequalities are clear.

THEOREM 2.6. Let $G$ be a finitely generated abelian group, then $R$ is finite dimensional if and only if $R G$ is finite dimensional. If $H$ is the torsion subgroup of $G$, then $\operatorname{dim} R \leqq \operatorname{dim} R G \leqq \operatorname{dim} R \cdot|H|$.

Proof. If $G$ is a finitely generated abelian group then $G \cong G_{1} \oplus G_{2} \oplus \cdots$ $\oplus G_{n} \oplus H$ where $|H|<\infty$ and $G_{i}$ for $1 \leqq i \leqq n$ is an infinite cyclic group. As in [2, p. 673], we define $A_{1}=R G_{1}, A_{2}=A_{1} G_{2}, \cdots, A_{n}=A_{n-1} G_{n}$, and $A=A_{n} H$; clearly $R G \cong A$. By Corollary 2.4 and Lemma 2.5 , we see by induction that $A$ is finite dimensional and consequently $R G$ is finite dimensional. The converse and inequalities follow easily.

3. Applications. Let $Z(R)$ denote the right singular ideal of $R$ (4).

Lemma 3.1. Let $G$ be a free abelian group, then $Z(R G)=Z(R) G$.

Proof. The proof uses the same technique as the proof of Theorem 2.7 of [10].

Proposition 3.2 (Connell, [2]). The group ring $R G$ is semiprime if and only if $R$ is semiprime and the order of no finite normal subgroup is a zero-divisor in $R$.

Proof. See the appendix of [4]. 
It is well known that a semiprime Goldie ring is a semiprime, finite dimensional ring with zero singular ideal.

COROLlaRY 3.3. Let $G$ be a free abelian group. A ring $R$ is a semiprime Goldie ring if and only if $R G$ is a semiprime Goldie ring.

Proof. The proof is immediate.

Proposition 3.4 (Burgess, [1]). If $Z(R G)=0$, then $Z(R)=0$ and the order of every finite normal subgroup of $G$ is a nonzero-divisor in $R$.

Proof. See Theorem 4.8 of [1].

A locally normal group is one in which every finite subset is contained in a finite normal subgroup.

Proposition 3.5 (Burgess, [1]). Assume that $G$ is locally normal and the order of every finite normal subgroup of $G$ is a nonzero-divisor in $R$. If $Z(R)=0$, then $Z(R G)=0$.

Proof. See 4.9 of [1].

COROLlaRY 3.6. Let $G$ be a finitely generated abelian group. Then $R$ is a semiprime Goldie ring and the order of every finite normal subgroup of $G$ is a nonzero-divisor in $R$ if and only if $R G$ is a semiprime Goldie ring.

Proof. The proof is immediate using the construction in the proof of Theorem 2.6.

A right ideal of a ring $R$ is said to be essential if it has nonzero intersection with every nonzero right ideal of $R$. A right ideal $D$ of $R$ is dense if for every $0 \neq r_{1} \in R$ and for every $r_{2} \in R$ there exists $r \in R$ such that $r_{1} r \neq 0$ and $r_{2} r \in D$. We denote the Jacobson radical of $R$ by $\operatorname{Rad} R$. A right ideal $A$ is said to be small if for every right ideal $B, A+B=R$ implies $B=R$. It is known that $A$ is small if and only if $A \subset \operatorname{Rad} R$.

The following remarks are well known.

Remark 3.7. A right ideal $D$ is dense in $R$ if and only if $D G$ is dense in $R G$.

RemarK 3.8. A right ideal $L$ is essential in $R$ if and only if $L G$ is essential in $R G$.

A right ideal $B$ is rationally closed in $R$ if $x^{-1} B=\{r \in R \mid x r \in B\}$ is not dense for all $x \in R-B$. Let $I(R)$ denote the injective hull of $R$, then $B$ is rationally closed in $R$ if there exists a subset $S$ of $I(R)$ such that $B=$ $\{x \in R \mid S x=0\}$ [8].

LEMMA 3.9. A right ideal $K$ of $R$ is rationally closed in $R$ if and only if $K G$ is rationally closed in $R G$. 
Proof. If $K$ is rationally closed then there exists a subset $S \subset I(R)$ such that $K=\{x \in R \mid S x=0\}$. We will show that $K G=\{x \in R G \mid S G x=0\}$. Let $x \in K G$ then $S G x=0$ since $S k=0$ for all $k \in K$. Hence $x \in\{x \in R G \mid$ $S G x=0\}$. Now suppose $0 \neq x \notin K G$. We want to show there exists $y \in S G$ such that $y x \neq 0$. Let $x=r_{1}\left(g_{1}\right) g_{1}+\cdots+r_{1}\left(g_{n}\right) g_{n}$, since $x \notin K G$ there exists $r_{i}\left(g_{i}\right)$ such that $r_{i}\left(g_{i}\right) \notin K . K$ is rationally closed so there exists $0 \neq s \in S$ such that $s r_{i}\left(g_{i}\right) \neq 0$. Hence, $s x \neq 0$ implies $x \notin\{x \in R G \mid S G x=0\}$.

Conversely, suppose $K$ is not rationally closed in $R$, then there exists $x \in R-K$ such that $x^{-1} K$ is dense in $R$. Thus $\left(x^{-1} K\right) G=x^{-1} K G$ is dense in $R G$ and hence $K G$ is not rationally closed in $R G$.

Proposition 3.10 (Renault, [6]). The group ring $R G$ is self-injective if and only if $R$ is self-injective and $G$ is finite.

Proof. See [6].

Lemma 3.11 (SHock, [9]). Let $R$ be a self-injective ring. Then $R$ is a cogenerator if and only if $R$ is right finite dimensional and $Z(R)$ is rationally closed.

Proof. See Proposition 2 of [9].

If $R$ is a self-injective ring then $Z(R)=\operatorname{Rad} R$ [4]. It is known that if $R$ is self-injective and finite dimensional then $R / \operatorname{Rad} R$ is completely reducible.

THEOREM 3.12. Let $G$ be a finite group where the order of $G$ is a unit in $R$, then $R$ is a self-injective cogenerator ring if and only if $R G$ is a selfinjective cogenerator ring.

Proof. Let $R$ be a self-injective cogenerator ring. It is clear that $R G$ is finite dimensional and injective. By Lemma 3.11, we need only show that $Z(R G)$ is rationally closed. It is clear that if $R$ contains no proper dense right ideals then every right ideal is rationally closed and conversely. So, we shall show that $R G$ contains no proper dense right ideals. Let $D$ be a dense right ideal of $R G$. Then $D+Z(R) G$ is dense and by Proposition 5.1 of [8], $(D+Z(R) G) / Z(R) G$ is dense in $R G / Z(R) G$ since $Z(R) G$ is rationally closed. Clearly, $R G / Z(R G)$ and $R / Z(R)$ are completely reducible. Therefore, $(R / Z(R)) G \cong R G / Z(R) G$ is completely reducible [2] and thus $R G / Z(R) G$ contains no proper dense right ideals. Hence, $D+Z(R) G=R G$. But $Z(R) G \subset Z(R G)=\operatorname{Rad} R G$ implies $Z(R) G$ is small. Hence, $D=R G$.

Conversely, let $D$ be dense in $R, D \neq R$, then $D G$ is dense in $R G$ and $D G \neq R G$. 
Lemma 3.13 (Shock, [9]). Suppose that $Z(Q(R))$ is the Jacobson radical of $Q(R)$ and is rationally closed. If $Q(R) / Z(Q(R))$ is a completely reducible ring and $R / Z(R)$ is semiprime, then $R$ is a right order in $Q(R)$.

Proof. See Proposition 4 of [9].

THEOREM 3.14. Let $G$ be a finite group, then $R$ is a right order in a self-injective cogenerator ring and the order of no finite normal subgroup of $G$ is a zero-divisor in $R$ if and only if $R G$ is a right order in a self-injective cogenerator ring.

Proof. Let $R$ be a right order in a self-injective cogenerator ring $Q$, then $Q=Q(R)$. By 3.6 of [1], we have $Q(R G) \cong Q(R) G$ and thus by Theorem $3.12 Q(R G)$ is a self-injective cogenerator ring. It is now clear that both $Q(R G) / Z(Q(R G))$ and $Q(R) / Z(Q(R))$ are completely reducible. Also, it is clear that $Q(R) G / Z(Q(R)) G$ is completely reducible and that $R G / Z(R) G$ is semiprime. By Lemma 3.13 we need only to show that $R G / Z(R G)$ is semiprime. To do this, we first show that $Z(R) G=Z(R G)$. It is sufficient to show that $Z(Q(R G))=Z(Q(R)) G$ since $Z(R G)=Z(Q(R G)) \cap R G=$ $Z(Q(R) G) \cap R G=Z(Q(R)) G \cap R G=Z(R) G$. Now $(Q(R) /(Z(Q(R)))) G \cong$ $Q(R) G / Z(Q(R)) G \cong Q(R G) / Z(Q(R)) G$. Recall $Z(Q(R)) G \subseteq Z(Q(R G))=$ $\operatorname{Rad} Q(R G)$. Hence, $Z(Q(R)) G=Z(Q(R G))$ since $Q(R G) / Z(Q(R)) G$ is completely reducible. The converse follows similarly.

In [12] Smith showed that if $G$ is a poly- (cyclic or finite) group and $R$ is a right order in a right Artinian ring then $R G$ is a right order in a right Artinian ring. We extend this result to a class of group rings, where $G$ need not be poly- (cyclic or finite), using a method of Small [11].

THEOREM 3.15. Let $G$ be a free abelian group. If $R$ is a right order in a right Artinian ring then $R G$ is a right order in a right Artinian ring.

Proof. It is clear that $\operatorname{rad}(R G)=(\operatorname{rad} R) G$ when $G$ is free abelian. We now use the same argument as in Theorem 3.6 of [10].

\section{REFERENCES}

1. W. D. Burgess, Rings of quotients of group rings, Canad. J. Math. 21 (1969), 865-875. MR 39 \#5714.

2. I. G. Connell, On the group ring, Canad. J. Math. 15 (1963), 650-685. MR 27 \#3666.

3. A. W. Goldie, Semiprime rings with maximum condition, Proc. London Math. Soc. (3) 10 (1960), 201-220. MR 22 \#2627.

4. J. Lambek, Lectures on rings and modules, Blaisdell, Waltham, Mass., 1966. MR 34 \#5857.

5. D. S. Passman, Infinite group rings, Dekker, New York, 1971.

6. G. Renault, Sur les anneaux de groupes, C. R. Acad. Sci. Paris Sér. A-B 273 (1971), A84-A87. MR 44 \#5387. 
7. P. Ribenboim, Rings and modules, Interscience Tracts in Pure and Appl. Math., no. 24, Interscience, New York, 1969. MR 39 \#4204.

8. R. C. Shock, Injectivity, annihilators, and orders, J. Algebra 19 (1971), 96-103. MR 43 \#4861.

9. - Orders in self-injective cogenerator rings, Proc. Amer. Math. Soc. 35 (1972), 393-398.

10. _ Polynomial rings over finite dimensional rings, Pacific J. Math. 42 (1972), 251-258.

11. L. W. Small, Orders in Artinian rings. II, J. Algebra 9 (1968), 266-273. MR 37 \#6315.

12. P. F. Smith, Quotient rings of group rings, J. London Math. Soc. 3 (1971), 645-660.

Department of Mathematics, Southern Illinois University, Carbondale, ILLINOIS 62901 